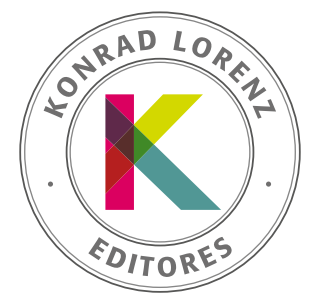

\title{
Intolerancia a la frustración y estrategias cognitivas de regulación emocional en la predicción de la agresividad
}

\author{
Leonardo A. Medrano ${ }^{\mathrm{a}, \mathrm{b}}$, Paul Franco ${ }^{\mathrm{c}}$, Pablo E. Flores-Kanter ${ }^{\mathrm{a}, \mathrm{b}}$ y Alba E. Mustacac,
}

a Universidad Siglo 21, Córdoba, Argentina

${ }^{b}$ Universidad Nacional de Córdoba, Argentina

c Universidad Abierta Interamericana (UAI), CABA, Argentina

Recibido el 1 de noviembre de 2018; aceptado el 26 de marzo de 2019

\author{
PALABRAS CLAVE \\ Estrategias cognitivas \\ de regulación \\ emocional, \\ intolerancia a \\ la frustración, \\ agresividad
}

\section{KEYWORDS}

Cognitive emotional regulation strategies, frustration intolerance, aggressiveness

\begin{abstract}
Resumen La conducta agresiva constituye un problema que afecta a los individuos y a la sociedad. El Modelo de Agresión General integra un conjunto de variables que influyen en la probabilidad de exhibir una conducta agresiva entre las cuales está la regulación emocional y la frustración. Esta investigación evalúa las relaciones entre la regulación emocional cognitiva y la intolerancia a la frustración. A 419 sujetos adultos se les administraron la Escala de Intolerancia a la Frustración, la Escala de Regulación Emocional Cognitiva y el Cuestionario de Agresión. Se hallaron relaciones positivas en la mayoría de las estrategias desadaptativas cognitivas de regulación emocional y relaciones negativas entre la mayoría de las estrategias cognitivas adaptativas de regulación emocional con la conducta agresiva, y relaciones positivas entre todas las dimensiones de la intolerancia a la frustración y la conducta agresiva. El Análisis de Regresión Múltiple muestra que las variables que producen un cambio significativo en la agresividad son la catastrofización y culpar a otros, así como la intolerancia a la frustración referida a derechos. Mediante path análisis se verificó que estas tres variables mencionadas permiten explicar un $41 \%$ de la variabilidad en la agresividad.

(c) 2019 Fundación Universitaria Konrad Lorenz. Este es un artículo Open Access bajo la licencia CC BY-NC-ND (http://creativecommons.org/licenses/bync-nd/4.0/).
\end{abstract}

Frustration intolerance and cognitive strategies of emotional regulation in the prediction of aggressiveness

Abstract Aggressive behavior is a problem that affects individuals and society. The General Aggression Model integrates a set of variables that influence the probability of exhibiting aggressive behavior, among which is emotional regulation and frustration. This research assesses the relationships between cognitive emotional regulation and frustration intolerance. A total of 419 adult subjects were administered the Frustration Intolerance Scale, the Cognitive Emotional Regulation Scale and the Aggression Questionnaire. Positive relationships were

* Autor para correspondencia.

Correo electrónico: albamustaca@gmail.com 
found in most of the maladaptive cognitive emotional regulation strategies and negative relationships between most of the adaptive cognitive emotional regulation strategies with the aggressive behavior, and positive relationships between all the dimensions of the frustration intolerance and the aggressive behavior. The Multiple Regression Analysis shows that the variables that produce a significant change in aggressiveness are catastrophization and blaming others, as well as the frustration intolerance referring to rights. By means of path analysis, it was verified that these three variables can explain $41 \%$ of the variability in aggressiveness.

(c) 2019 Fundación Universitaria Konrad Lorenz. This is an open access article under the CC BYNC-ND license (http://creativecommons.org/licenses/bync-nd/4.0/).

La agresividad es uno de los componentes de la respuesta agonística que se encuentra presente en todas las especies; su expresión debe modularse para asegurar el éxito de los individuos, grupos y sociedades. El tema es abordado por muchas disciplinas, que incluye, entre otras, a la sociología, antropología, biología y psicología. Actualmente adquiere más relevancia, ya que la violencia desmedida es un problema que hay que abordar con técnicas eficaces para lograr un mayor bienestar en los individuos y en la sociedad.

Si bien existen muchas definiciones del comportamiento agresivo (CA), la mayoría de ellas tienen al menos tres elementos en común: (1) intencionalidad: el que la emite tiene en general una meta, (2) tiene consecuencias aversivas hacia el que la recibe y (3) se manifiesta de muchas maneras, estableciéndose una amplia variedad de tipologías del CA (Anderson \& Bushman, 2001, 2002; Geen, 2001). En este sentido, una de las escalas autoadministradas más usadas para evaluar la agresividad es el Cuestionario de Agresividad de Buss y Perry (1992), quienes a través de análisis factoriales, la categorizaron en cuatro tipos: agresión física, agresión verbal, ira y hostilidad.

En cuanto a las teorías de la conducta agresiva, la más aceptada es el Modelo de Agresión General (GAM) (Anderson \& Bushman, 2002) que integra a las cuatro principales teorías preexistentes que presentaron evidencia: (1) las instintivas, que considera que el CA está determinado biológicamente (e.g., Freud, 1992; Lorenz, 1966); (2) las reactivas, que la considera causada por una activación generalizada que predomina por algún evento como la frustración (e.g., Berkowitz, 1989; Dollard, Dobb, Miller, Mower \& Sears, 1939); (3) las psicosociales, que la considera como un conjunto de patrones de respuesta aprendidos por condicionamiento clásico, operante (Skinner, 1953) o de manera vicaria (e.g., Bandura, 1999, De la Torre-Luque \& ValeroAguayo, 2013; Wiedeman, Black, Dollea, Finney \& Coker, 2015) y (4) las cognitivas, que se centran en la conducta encubierta, como el pensamiento, las creencias y los valores que se ubican entre el estímulo y la respuesta (e.g., Archer \& Haigh, 1997; Beck, 2003; Beck \& Freeman, 1995).

El modelo GAM se centra en tres variables que interaccionan entre sí: las entradas, las internas y el resultado. Las entradas son factores relacionados con la situación y el individuo. Los situacionales son eventos externos que aumentan la probabilidad de la agresión: provocaciones, insultos, consumo de drogas, exposición a conductas agresivas, etc. Las variables individuales incluyen patrones biológicos o genéticos: personalidad, género, creencias, valores, etc. Estos factores influyen en los estados del individuo, clasificados en cognitivos, afectivos y fisiológicos. Lo cognitivo implica el acceso hacia el pensamiento de hostilidad o agresividad; lo afectivo incluye el estado de ánimo negativo, humor, etc.; lo fisiológico es la activación o arousal medido por pulsaciones, ritmo cardíaco, etc., que aumenta la probabilidad de un comportamiento agresivo (CA). La tercera variable del modelo se refiere al resultado. Incluyen evaluaciones inmediatas, son casi automáticas, y una segunda instancia de reevaluaciones que ocurren cuando el resultado de la primera evaluación es insatisfactoria; son más conscientes, requiere más esfuerzo y recursos cognitivos y pueden conducir a un resultado más reflexivo. Todas estas variables interactúan entre sí y contribuyen a que aumenten o disminuyan las probabilidades de la expresión de un CA desproporcionado.

El GAM proporciona un marco teórico de referencia para realizar investigaciones que evalúen las probabilidades que, sobre determinadas variables, pueden predecir el CA. En este artículo presentaremos un trabajo que indaga si la regulación emocional (RE) y la intolerancia a la frustración (IF) predicen la propensión a un CA.

La RE se define como todo proceso explícito o implícito de naturaleza biológica, social, conductual y cognitiva que permite monitorear, evaluar y modificar una reacción emocional, cambiando su intensidad, duración o expresión (Denny, Silvers \& Ochsner, 2009; Garnefski \& Kraaij, 2007; Werner \& Gross, 2009). La RE puede ser deliberada y controlada e implica un esfuerzo consciente (Mauss, Bunge \& Gross, 2007; Mauss, Cook \& Gross, 2007), como, por ejemplo, inhibir una conducta de enojo en un conflicto interpersonal; o automática e inmediata, como cambiar el foco atencional de una imagen angustiante (Gross \& Thompson, 2007). Aunque hay evidencia que los procesos automáticos son aspectos importantes de la RE y pueden, incluso, ser ventajosas para la RE deliberada (Williams, Bargh, Nocera \& Gray, 2009), la mayoría de las investigaciones se centraron en estudiar la RE consciente (Mauss et al., 2007). Además, de acuerdo con los estudios sobre aprendizaje, está documentado que tácticas aprendidas deliberadamente se pueden transformar en automáticas, y viceversa (e.g., Anderson, 1982; Hayes \& Hofmann, 2018; Schneider \& Shiffrin, 1977).

Las líneas de investigación más productivas en el estudio de la RE son las desarrolladas por Garnefski y el grupo de Kraaij que se enfocaron en la medición de la RE de sucesos percibidos como estresantes para el sujeto (Garnefski \& Kraaij, 2007; Garnefski, Kraaij \& Spinhoven, 2001, 2002). Garnefski y Kraaij (2007) distinguieron nueve estrategias de RE cognitivas que las personas utilizan tras experimentar un evento estresante: (1) "Autoculparse", son pensamientos donde la persona se echa la culpa por lo vivenciado; (2) "Culpar a otros", pensamientos de echar la culpa a factores ambientales o a otras personas; (3) "Rumiación", que se 
refiere a tener pensamientos sobre las emociones e ideas asociadas al evento estresante; (4) "Catastrofización", pensamientos que magnifican lo negativo de lo que se ha experimentado; (5) "Poner en perspectiva pensamientos" que minimizan la gravedad de la situación; (6) "Refocalización positiva", uso de pensamientos respecto a temas placenteros o divertidos en lugar de centrarlos en el evento estresante; (7) "Reinterpretación positiva", usar pensamientos con connotación positiva dándole a lo sucedido un significado en términos de crecimiento personal; (8) "Aceptación”, son pensamientos de aceptación de lo acontecido, y (9) “Refocalización en los planes", se piensa en qué pasos seguir para resolver la situación problemática. Estas estrategias sirvieron de base para elaborar el Cuestionario de Regulación Emocional Cognitiva (CERQ) (Garnefski \& Kraaij, 2007), que se utilizará en el presente trabajo. Algunos autores consideran que estas estrategias requieren de recursos cognitivos subyacentes, como la conciencia emocional y la aceptación (Berking \& Znoj, 2008; Gratz \& Roemer, 2004; Gratz \& Tull, 2010; Greenberg, Elliott \& Pos, 2007).

Si bien no hay una forma de RE intrínsecamente adaptativa o desadaptativa (Gratz \& Roemer, 2004; Gross, 2002), hay algunas que se consideran más adecuadas que otras por los resultados obtenidos en diversas investigaciones. En este sentido, se propusieron modelos y se agruparon en cinco adaptativas: aceptación, refocalización positiva, refocalización en los planes, reinterpretación positiva y poner en perspectiva; y en cuatro desadaptativas: autoculparse, rumiación, catastrofización y culpar a otros (DomínguezSánchez, Lasa-Aristu, Amor \& Holgado-Tello, 2013; d'Acremont \& Van der Linden, 2007; Jermann, Van der Linden, d'Acremont \& Zermatten, 2006).

En relación con la agresión y la $\mathrm{RE}$, en niños y adolescentes se halló que las RE mal adaptativas están asociadas a un aumento de la agresión (Cohn, Jakupcak, Seibert, Hildebrandt \& Zeichner, 2010; McLaughlin, Hatzenbuehler, Mennin \& Nolen-Hoeksema, 2011; Roll, Koglin \& Petermann, 2012; Tager, Good \& Brammer, 2010; Tull, Jakupcak, Paulson \& Gratz, 2007). Un estudio longitudinal halló que los niños(as) con desregulación emocional se asoció a un comportamiento agresivo en la edad adolescente (Roll et al., 2012). Cohn et al. (2010), utilizando estudiantes universitarios, encontraron que la dificultad en la RE se asoció con aplicar choques eléctricos de mayor intensidad a un oponente ficticio. Roberton, Daffern y Bucks (2012) en una revisión informaron que las personas que subregulan la ira y otras emociones negativas tienen más probabilidades de comportarse agresivamente. A la vez, la sobrerregulación de la emoción puede llevar también a un CA. Finalmente, Roberton et al. (2012) concluyen que para lograr una RE deliberada se necesitan tres habilidades previas: conciencia, aceptación emocional y competencia en una variedad de tácticas de RE.

El segundo constructo que se evaluará en la predicción de la agresividad en este artículo es la IF. La frustración se define como un estado del organismo análogo al estrés y al dolor sensorial desencadenado ante la devaluación, omisión o inaccesibilidad inesperada de un reforzador positivo (Amsel, 1992; Mustaca, 2013). La hipótesis según la cual la frustración causa agresión la desarrollaron Dollard et al. (1939) y muchas investigaciones la confirmaron (e.g., Davis \& Donenfeld, 1967), y otros la refutaron (Mustaca, 2018;
Mustaca, Martínez \& Papini, 2000). Ante estos resultados, Berkowitz (1989) y Amsel (1992) consideraron que la presencia de agresión a causa de una frustración sería producto de una activación generalizada (fisiológica y emocional), que solo emerge cuando en un sujeto es la primera en su jerarquía de respuestas posibles. De esto se puede inferir que aquellas personas que tienen mayor IF serían las más propensas a exhibir un CA.

De lo revisado no se hallaron trabajos que evalúen simultáneamente la IF y la RE con la agresividad. De acuerdo con lo presentado anteriormente, se puede predecir que habrá una correlación positiva entre la IF y los tipos de RE no adaptativos y la agresividad, así como una correlación negativa entre la IF y los tipos de RE adaptativos con el CA. Además, siguiendo el modelo GAM y las teorías cognitivas de la ira (Beck, 2000; Deffenbacher, 2011; Harrington, 2005, 2007; Wranik \& Scherer, 2010), se pondrá a prueba un modelo explicativo de la agresividad mediante path análisis que podrá evaluar qué dimensiones de IF y de estrategias de RE tendrán más peso para predecir el CA.

\section{Método}

\section{Participantes}

Se tomó una muestra de 493 argentinos seleccionados de manera accidental, radicados principalmente en la Ciudad Autónoma de Buenos Aires (95\%). Las edades estuvieron comprendidas entre los 14 y los 80 años $(M=29.94, D E=12.95)$. En cuanto al sexo, $60.2 \%$ eran mujeres. En cuanto al nivel de estudios, el $0.4 \%$ no completó el primario, el 31.8\% tenía el secundario incompleto, el $14.5 \%$ el secundario completo, el $28.3 \%$ terciario o universitario incompleto, el $20.6 \%$ terciario o universitario completo, y el $4.5 \%$ posgrado. La mayoría de la muestra no estaba ni en tratamiento psicológico $(86.2 \%)$ ni psiquiátrico $(98.4 \%)$.

\section{Instrumentos}

Cuestionario de Agresión (CA) (Buss \& Perry, 1992), versión en castellano validada por Andreu, Peña y Graña (2002). Es autoadministrado, cuenta con 29 ítems respondidos en una escala tipo Likert de cinco puntos (1: "completamente falso para mí" a 5: "completamente verdadero para mí"). En la presente investigación la consistencia interna de la escala total, compuesta por las subescalas de agresividad física, verbal e ira, mostró valores óptimos $(a=.88)$.

Escala de Intolerancia a la Frustración (EIF) (The Frustration Discomfort Scale, Harrington, 2005), versión castellana (Medrano, Franco \& Mustaca, 2018). La versión original consta de 28 ítems que los sujetos deben responder utilizando una escala Likert de cinco categorías que van desde "No es nada característico de mí" (1), hasta "Es muy característico de mí" (5); a mayor puntaje mayor IF. La EIF tiene cuatro factores: F1: "Intolerancia a la incomodidad" (INCO, $a=.87)$; F2: “Derechos" (DE, $a=.85)$; F3: "Intolerancia emocional” (EMO, $a=.88)$, “Logro” (LO, $a=.84$ ) y el puntaje total (IFT, $a=.90)$. La escala argentina consta de 17 ítems distribuidos en los mismos factores que la escala original, presentando valores de consistencia interna aceptables ( $a$ entre .55 y .84), que fueron similares a la presente muestra. 
Escala de Regulación Emocional Cognitiva (ERE, versión castellana de Medrano, Moretti, Ortiz \& Pereno, 2013). Es autoadministrado, compuesto por 36 ítems respondidos en una escala Likert (1 es "casi nunca" y 5, "casi siempre”). Evalúa nueve estrategias de RE: cuatro desadaptativas (Culpar a otros, Autoculparse, Rumiación, Catastrofización) y cinco adaptativas (Aceptación, Poner en perspectiva, Reinterpretación positiva, Focalización en los planes, Focalización positiva). La consistencia interna en el presente estudio mostró valores a entre .61 y .71 en las dimensiones disfuncionales, y entre .64 y .84 en las funcionales.

\section{Procedimiento}

Se utilizó un diseño ex post facto retrospectivo, de un grupo y con múltiples medidas (Montero \& León, 2007). La recolección de los datos se llevó a cabo de manera presencial $(80.1 \%)$ y online. La participación fue voluntaria y antes de responder el cuestionario los participantes dieron su consentimiento informado.

\section{Análisis de datos}

Se realizaron análisis de regresión múltiple y un path análisis mediante modelos de ecuaciones estructurales. Las variables independientes ingresadas en el modelo de regresión correspondieron a las estrategias de la RE y las dimensiones de IF. La variable dependiente fue el CA. Previo al análisis de regresión múltiple se analizaron las correlaciones bivariadas, mediante coeficiente de Pearson, entre las diferentes variables incluidas en el modelo de regresión. Para el caso del path análisis se tuvieron consideraciones tanto teóricas como estadísticas para el diseño del modelo estructural. En cuanto al criterio estadístico, el modelo estructural atendió a los análisis de regresión previos efectuados e incluyó aquellas variables que habían obtenido coeficientes de regresión estadísticamente significativos para la predicción de la agresividad. El modelo puesto a prueba incluyó variables que desde el plano teórico se consideran más ligadas con la agresividad y la ira. Concretamente, se atendieron a las teorías cognitivas de la agresividad y la ira, que mencionan tres factores claves en la predicción de esta emoción: el culpar a otros, la catastrofización y las creencias irracionales de IF referida a derechos (Beck, 2000; Deffenbacher, 2011; Harrington, 2005, 2007). Para evaluar el ajuste del modelo propuesto se llevaron a cabo las estimaciones de los parámetros mediante el método de máxima verosimilitud. Siguiendo la literatura especializada (Hu \& Bentler, 1999; Lei \& Lomax, 2005), se utilizaron los índices chi cuadrado $\left(\mathrm{x}^{2}\right)$, comparative fit index (CFI), tucker lewis index (TLI), goodness of fit index (GFI) y root mean square error of approximation (RMSEA). Como proponen Hu y Bentler (1999) y Byrne (2010), se consideran aceptables valores comprendidos entre .05 y .08; para el índice RMSEA se consideran óptimos los valores menores a .05; aceptables valores superiores a .90 para los índices CFI, TLI y GFI, y óptimos valores que superiores o igual a .95. Para llevar a cabo los análisis mencionados se utilizó el programa AMOS IBM 20, y el software SPSS IBM 20.

\section{Resultados}

\section{Correlaciones preliminares}

En las tablas 1, 2 y 3 se detallan las correlaciones bivariadas para las respectivas variables que luego serán incluidas en el modelo de regresión múltiple.

Se observan relaciones positivas significativas entre la mayoría de las estrategias cognitivas desadaptativas de RE y CA. Las correlaciones con la agresividad de mayor intensidad se dan con la catastrofización $(r=.31 ; p<.01)$ y culpar a otros $(r=.31 ; p<.01)$.

Tabla 1 Correlación entre CA y RE desadaptativas

\begin{tabular}{lccccc}
\hline & Agresividad & Rumiación & Catastrofización & Autoculparse & Culpar a otros \\
\hline Agresividad & 1 & .02 & $.31^{* *}$ & $.10^{* *}$ & $.31^{* *}$ \\
Rumiación & & 1 & $.26^{* *}$ & $.35^{* *}$ & .08 \\
Catastrofización & & & 1 & $.32^{* *}$ & $.39^{* *}$ \\
Autoculparse & & & & 1 & .06 \\
Culpar a otros & & & & & 1 \\
\hline
\end{tabular}

Nota. ${ }^{*} p<.05,{ }^{* *} p<.01$.

Tabla 2 Correlación entre CA y RE adaptativas

\begin{tabular}{|c|c|c|c|c|c|c|}
\hline & Agresividad & Aceptación & F. Positiva & Perspectiva & Reinterpre. & R. planes \\
\hline Agresividad & 1 & $-.09^{*}$ & $-.15^{* *}$ & -.05 & $-.17^{* *}$ & -.03 \\
\hline Aceptación & & 1 & $.21^{* *}$ & $.36^{* *}$ & $.38^{* *}$ & $.37^{* *}$ \\
\hline F. Positiva & & & 1 & $.35^{\star *}$ & $.53^{\star *}$ & $.29^{* *}$ \\
\hline Perspectiva & & & & 1 & $.41^{* *}$ & $.30^{* *}$ \\
\hline Reinterpre. & & & & & 1 & $.50^{* *}$ \\
\hline R. planes & & & & & & 1 \\
\hline
\end{tabular}

Nota. ${ }^{*} p<.05,{ }^{* *} p<.01 ; \mathrm{F} .=$ Focalización, Reinterpre. $=$ Reinterpretación, R. =Refocalización. 
Se aprecian relaciones negativas estadísticamente significativas entre la mayoría de las estrategias cognitivas adaptativas de RE y CA. Las correlaciones con la agresividad de mayor intensidad se dan con la focalización positiva $(r=-.15 ; p<.01)$ y reinterpretación $(r=-.17 ; p<.01)$.

Se observan relaciones positivas significativas entre todas las dimensiones de la IF y el CA; la correlación entre la agresividad y la dimensión de derechos presenta una magnitud claramente mayor $(r=.48 ; p<.01)$.

\section{Análisis de regresión múltiple}

Al ingresar al modelo de regresión, las variables independientes correspondientes a las estrategias de RE y las dimensiones de IF tomando como variable dependiente el CA se encuentra que las únicas variables que producen un cambio significativo en el CA son la catastrofización $(\beta=.10$, $p=.02)$ y culpar a otros $(\beta=.16, p<.01)$, así como la intolerancia referida a derechos $(\beta=.45, p<.01)$. El modelo de regresión especificado permite explicar un $31 \%(p<.01)$ de la variabilidad en la agresividad (véase tabla 4 ).

\section{Análisis de ecuaciones estructurales: path análisis}

Dado que se utilizó una versión no validada al contexto argentino del instrumento de agresividad, en primer lugar se verificó el ajuste del modelo de medida para dicha variable latente (Byrne, 2010). El modelo de agresividad compuesto por los indicadores de agresividad verbal (ítems 1, 5, 17, 21, 27), física (ítems 2, 6, 10, 14) e ira (ítems 3, 7, $11,19,22,25)$ muestra un ajuste adecuado $\left(x^{2}=5.62, p=.46\right.$, $\mathrm{CFI}=.94, \mathrm{TLI}=.93, \mathrm{GFI}=.95$, RMSEA=.05).

Corroborado el modelo de medida se procedió a verificar el modelo estructural. En la figura 1 se observan los coeficientes estandarizados de regresión obtenidos para el modelo, y el coeficiente de determinación resultante para la variable CA.

El modelo presenta un ajuste adecuado $\left(\chi^{2}=283.33\right.$, $\mathrm{gl}=130, \mathrm{CFI}=.93, \mathrm{TLI}=.92, \mathrm{GFI}=.94, \mathrm{RMSEA}=.05)$ que explica en gran medida los componentes de la agresividad $\left(R^{2}=.41\right)$. Todos los paths del modelo son estadísticamente significativos y en la dirección esperada teóricamente. Al evaluar

Tabla 3 Correlación entre CA y IF

\begin{tabular}{|c|c|c|c|c|c|}
\hline & Agresividad & I. incomodidad & I. derechos & I. emocional & I. logro \\
\hline Agresividad & 1 & $.17^{* *}$ & $.48^{* *}$ & $.17^{* *}$ & $.12^{* *}$ \\
\hline I. incomodidad & & 1 & $.40^{* *}$ & $.31^{* *}$ & $.13^{* *}$ \\
\hline I. derechos & & & 1 & $.43^{* *}$ & $.35^{* *}$ \\
\hline I. emocional & & & & 1 & $.35^{* *}$ \\
\hline I. logro & & & & & 1 \\
\hline
\end{tabular}

Nota. ${ }^{*} p<.05,{ }^{* *} p<.01 ;$ I. $=$ Intolerancia.

Tabla 4 Análisis de regresión múltiple

\begin{tabular}{lccccc}
\hline & \multicolumn{2}{c}{ Coeficientes no estandarizados } & Coeficientes estandarizados & & \\
\cline { 2 - 5 } & $B$ & $E . E$. & $\beta$ & $t$ & $p$ \\
\hline (Constante) & 29.76 & 4.53 & & 6.56 & $<.01$ \\
Rumiación & -.38 & .25 & -.07 & -1.54 & .12 \\
Catastrofización & .42 & .18 & .10 & 2.25 & .02 \\
Autoculparse & .18 & .22 & .03 & .82 & .40 \\
Culpar a otros & .78 & .20 & .16 & 3.89 & $<.01$ \\
Aceptación & .17 & .25 & .03 & .70 & .47 \\
Focalización positiva & -.27 & .16 & -.07 & -1.66 & .09 \\
Poner en perspectiva & -.21 & .18 & -.05 & -1.16 & .24 \\
Reinterpretación positiva & -.36 & .27 & -.07 & -1.32 & .18 \\
Refocalización en los planes & .12 & .26 & .02 & .48 & .62 \\
Intolerancia: incomodidad & -.26 & .22 & -.05 & -1.20 & .23 \\
Intolerancia: derecho & 1.33 & .13 & .45 & 9.60 & $<.01$ \\
Intolerancia: emocional & -.12 & .14 & -.03 & -.85 & .39 \\
Intolerancia: logro & -.32 & .22 & -.06 & -1.40 & .16 \\
\hline
\end{tabular}

Nota. Se destacan en negrita los coeficientes de regresión estadísticamente significativos. 


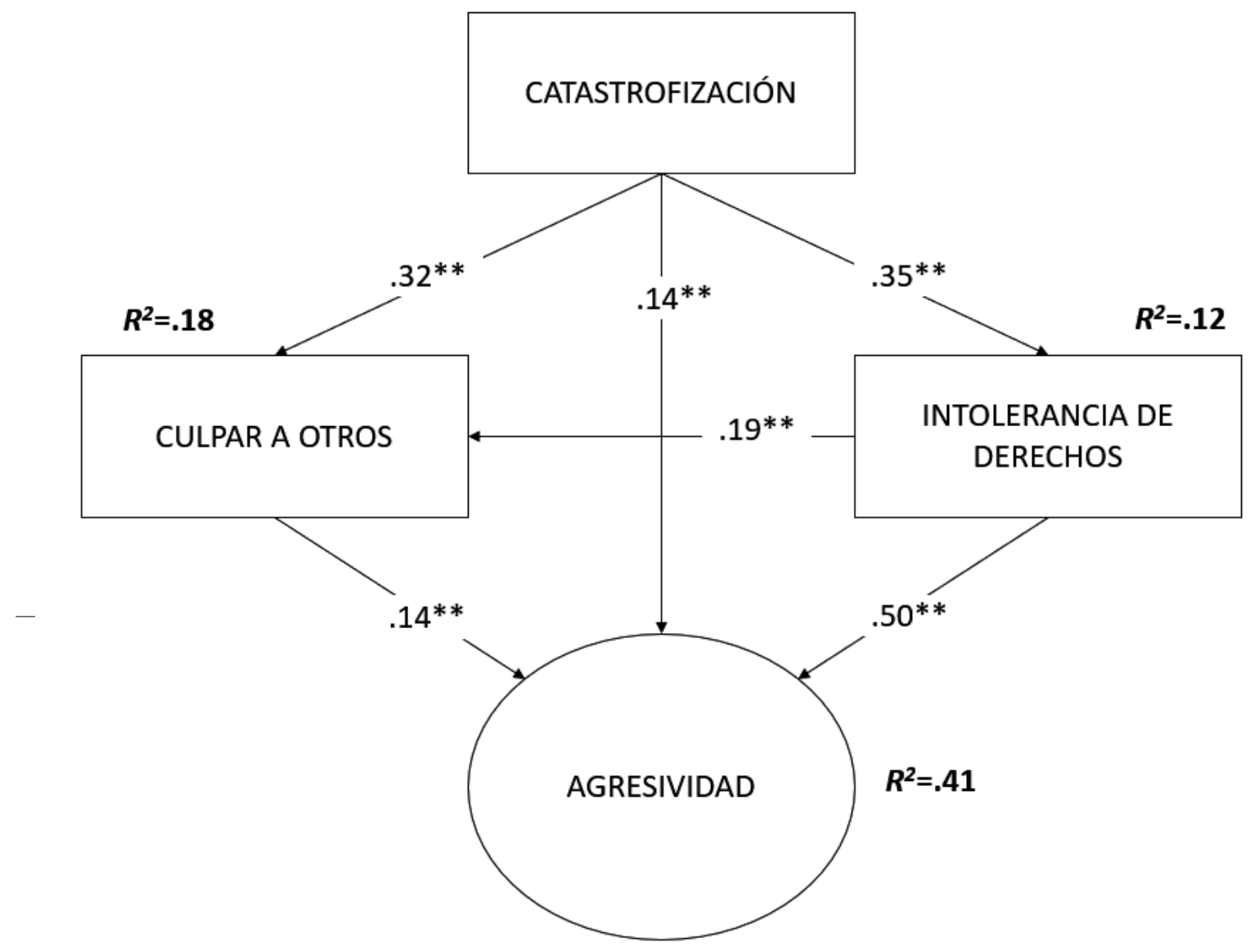

Figura 1 Path análisis: coeficientes estandarizados

Nota. ${ }^{* *} p<.01$.

los efectos totales de las respectivas variables la IF tiene un efecto total mayor $(\beta$ Total=.53), seguido por la catastrofización ( $\beta$ Total=.39), y el culpar a otros ( $\beta$ Total=.14). El efecto de la catastrofización sobre la agresividad se verifica principalmente de manera indirecta, a través de la moderación de la intensidad de las variables culpar a otros $(\beta=.32)$ e intolerancia referida a derechos $(\beta=.35)$. En cambio, en la IF el efecto más fuerte en la agresividad es el path directo sobre la misma $(\beta=.50)$.

\section{Discusión}

La agresividad es una emoción que conlleva grandes implicancias en la salud de los individuos. Esta investigación se propuso como objetivo central evaluar el poder de predicción de variables cognitivas sobre el CA. Concretamente, se indagó la capacidad predictiva de las estrategias de RE y de las creencias irracionales que producen mayor IF.

Se obtuvo que las variables que permiten explicar con mayor peso la agresividad son los factores de RE catastrofización y culpar a otros, y la IF referida a derechos. Esto resulta coherente con el modelo GAM, que hipotetiza que las variables cognitivas son factores claves en la generación de la agresividad, entendiéndolas como input o factores desencadenantes de ira (Deffenbacher, 2011; Ruddle, Pina \& Vásquez, 2017). El culpar a otros y la IF referida a derechos han sido contempladas también por los principales modelos cognitivos teóricos explicativos de la ira (Beck, 2000; Deffenbacher, 2011; Harrington, 2005, 2007; Wranik \& Scherer, 2010). El que la intolerancia a derechos sea la creencia más predominante en la predicción de la agresividad también va en línea con los postulados de la teoría racional emotiva de Ellis (véase Harrington, 2005, 2007).

Mediante el path análisis pudo evidenciarse que mientras la catastrofización moderaría la intensidad de la expresión de agresividad, afectando principalmente a culpar a otros (CO) e intolerancia de derecho (ID), estas últimas tienen un efecto directo sobre los componentes de la agresividad. Respecto al efecto moderador de la catastrofización, los resultados son coherentes con los postulados de Beck (2000) y Deffenbacher (2011), quienes plantean que la tendencia a sobregeneralizar la importancia del evento y sus consecuencias negativas modera la intensidad en que se experimenta la agresividad. Sobre este último punto, es importante también mencionar que la variable catastrofización, tal cual como es medida por el CERQ, solapa operacional y conceptualmente con la rumiación (Selby, Anestis \& Joiner, 2008). En efecto, refiere a la tendencia a pensar continuamente acerca de cuán mala es una situación y de los efectos negativos que la situación actual tendrá en el futuro. La rumiación es otro de los componentes cognitivos claves a la hora de explicar los comportamientos vinculados con la ira (Deffenbacher, 2011).

Desde un marco interdisciplinar o transdisciplinario en el abordaje teórico de la ira y la conducta agresiva, 
Potegal y Stemmler (2009) señalan que para comprender estos fenómenos es necesario contemplar distintos factores que interaccionan entre sí. En su revisión, estos autores indican que un desencadenante básico de la ira es la frustración, y entre ella, la referida al sentido de injusticia. Por su parte, la rumiación permite explicar el mantenimiento de la ira en el tiempo. Luego, el paso de sentirse molesto a la conducta agresiva se explicaría por una conexión particular entre las estructuras límbicas y la corteza orbitofrontal. En suma, los abordajes actuales de la conducta agresiva como el GAM intentan explicar estos comportamientos desde un punto de vista transdisciplinar.

Si bien los resultados presentados son coherentes con las investigaciones previas que han ligado ciertos componentes cognitivos con la ira, principalmente la catastrofización y el culpar a otros (Besharat, Nia \& Farahani, 2013; Martin \& Dahlen, 2005) y la intolerancia a derechos (Harrington, 2006; Jibeen, 2012, 2016; Stankovic' \& VukosavljevićGvozden, 2011; Tripaldi et al. 2018), estos antecedentes han estudiado las variables por separado. En este sentido, las investigaciones previas han hecho hincapié también en la necesidad de medir creencias relevantes como la intolerancia a derechos juntamente con otras variables como el culpar a otros, y su efecto conjunto en la ira (Stankovic' \& Vukosavljević-Gvozden, 2011). Si bien recientemente Rodríguez, Baker, Pu y Tucker (2017) presentaron evidencia sobre la relación entre la capacidad de regulación emocional, la intolerancia a la frustración y ciertas conductas agresivas, hasta la fecha, este es el primer estudio que permite ver cómo interactúan los factores cognitivos de regulación emocional junto con las creencias irracionales vinculadas a la intolerancia, a la frustración, y cómo, conjuntamente, predicen la agresividad.

Aun así, este estudio no carece de limitaciones. Principalmente, el tipo de diseño transaccional utilizado no permite sacar conclusiones definitivas respecto a la relación temporal entre las variables implicadas. Los modelos que implican relaciones de causalidad deben ser probados mediante diseños más acordes, longitudinales o experimentales, que permiten hacer inferencias de mayor rigurosidad sobre las relaciones temporales/causales implicadas (Cole \& Maxwell, 2003).

En conclusión, se presenta evidencia que sustenta los principales modelos cognitivos de la ira y el CA, destacando el aporte central de la IF y las dimensiones de cognitivas vinculadas a la RE.

\section{Agradecimientos}

Investigación financiada por la Universidad Siglo 21 y Universidad Abierta Interamericana. Se agradecen los comentarios de los revisores anónimos y a los editores que contribuyeron a mejorar la calidad del artículo.

\section{Referencias}

Amsel, A. (1992). Frustration theory. Cambridge, UK: Cambridge University Press. Appleton. Traducción al castellano en Madrid: Alianza, 1984. http://dx.doi.org/10.1017/CBO9780511665561

Anderson, J. R. (1982). Acquisition of cognitive skill. Psychological Review, 89, 369-406. http://dx.doi.org/10.1037/0033-295X.89.4.369.
Anderson, C. A., \& Bushman, B. J. (2001). Effects of violent video games on aggressive behavior, aggressive cognition, aggressive affect, physiological arousal, and prosocial behavior: A meta-analytic review of the scientific literature. Psychological Science, 12, 353-359. http://dx.doi.org/10.1111/1467-9280.00366

Anderson, C. A., \& Bushman, B. J. (2002). Human aggression. Annual Review Psychology, 53, 27-51. http://dx.doi.org/10.1146/ annurev.psych.53.100901.135231

Andreu, J. M., Peña, M. E., \& Graña, J. L. (2002). Adaptación psicométrica de la versión española del Cuestionario de Agresión. Psicothema, 14, 476-482.

Archer, J., \& Haigh, A. (1997). Do beliefs about aggression predict self-reported levels of aggression? British Journal of Social Psychology, 38, 71-84. http://dx.doi.org/10.1002/ab.10029

Bandura, A. (1999). Moral disengagement in the perpetration of inhumanities. Personality and Social Psychology Review, 3(3), 193209. http://dx.doi.org/10.1207/s15327957pspr0303_3

Beck A., \& Freeman, A. (1995). Terapia cognitiva de los trastornos de personalidad. Barcelona: Paidós.

Beck, A. (2000). Prisoners of hate: The cognitive basis of anger, hostility and violence. New York: Perennial.

Beck, A. (2003). Prisioneros del odio. Las bases de la ira, la hostilidad y la violencia. Barcelona: Paidós.

Berking, M., \& Znoj, H. (2008). Development and validation of a self-report measure for the assessment of emotion regulation skills (SEK-27). Zeitschrift Fur Psychiatrie Psychologie Und Psychotherapie, 56(2), 141-153. http//dx.doi.org/10.1024/1661-4747. 56.2.141

Berkowitz, L. (1989). Frustration-aggression hypothesis: Examination and reformulation. Psychological Bulletin, 106, 59-73. http://dx.doi.org/10.1037/0033-2909.106.1.59

Besharat, M. A., Nia, M. E., \& Farahani, H. (2013). Anger and major depressive disorder: The mediating role of emotion regulation and anger rumination. Asian Journal of Psychiatry, 6(1), 35-41. http://dx.doi.org/10.1016/j.ajp.2012.07.013

Buss, A. H., \& Perry, M. (1992). The aggression questionnaire. Journal of Personality and Social Psychology, 63, 452-459. http:// dx.doi.org/10.1037/0022-3514.63.3.452

Byrne, B. M. (2010). Structural equation modeling with AMOS ( $2^{\text {nd }}$ ed.). New York: Routledge.

Cohn, A. M., Jakupcak, M., Siebert, L. A., Hildebrandt, T. B., \& Zeichner, A. (2010). The role of emotion regulation in the association between men's restrictive emotionality and use of physical aggression. Psychology of men and Masculinity, 11(1), 53-64. http://dx.doi.org/10.1037/a0018090

Cole, D. A., \& Maxwell, S. E. (2003). Testing mediational models with longitudinal data: Questions and tips in the use of structural equation modeling. Journal of Abnormal Psychology, 112(4), 558-577. http://dx.doi.org/10.1037/0021-843x.112.4.558

Davis, H., \& Donenfeld, I. (1967). Extintion induced social interaction in rats. Psychonomic Science, 7, 85-86. http://dx.doi. org/10.3758/BF03328475

De la Torre-Luque, A., \& Valero-Aguayo. (2013). Factores moduladores de la respuesta agresiva tras la exposición a videojuegos violentos. Anales de Psicología, 29, 311-318. http://dx.doi. org/10.6018/analesps.29.2.132071

Deffenbacher, J. L. (2011). Cognitive-behavioral conceptualization and treatment of anger. Cognitive and Behavioral Practice, 18(2), 212-221. http://dx.doi.org/10.1016/j.cbpra.2009.12.004.

Denny, B. T., Silvers, J. A., \& Ochsner, K. N. (2009). How we heal what we don't want to feel: The functional neural architecture of emotion regulation. In A. M. Kring \& D. M. Sloan (Eds.), Emotion regulation and psychopathology: A transdiagnostic approach to etiology and treatment (pp. 59-87). New York: Guilford Press.

Dollard, J., Dobb, L. W., Miller, N. E., Mower, O. H., \& Sears, R. R. (1939). Frustration and agression. New Haven, CN: Yale University Press. 
d'Acremont, M., \& Van der Linden, M. (2007). How is impulsivity related to depression in adolescence? Evidence from a French validation of the cognitive emotion regulation questionnaire. Journal of Adolescence, 30(2), 271-282. http://dx.doi.org/10.1016/j.adolescence.2006.02.007

Domínguez-Sánchez, F., Lasa-Aristu, A., Amor, P., \& Holgado-Tello, F. (2013). Psychometric properties of the spanish version of the cognitive emotion regulation questionarie. Assessment, 20(2), 253-261. http://dx.doi.org/10.1177/1073191110397274

Freud, S. (1992). El malestar en la cultura y otros ensayos. Madrid: Alianza Editorial.

Garnefski, N., \& Kraaij, V. (2007). The cognitive emotion regulation questionnaire: Psychometric features and prospective relationships with depression and anxiety in adults. European Journal of Psychological Assessment, 23, 141-149. http://dx. doi.org/10.1027/1015-5759.23.3.141

Garnefski, N., Kraaij, V., \& Spinhoven, P. (2001). Negative life events, cognitive emotion regulation and emotional problems. Personality and Individual Differences, 30, 1311-1327. http://dx. doi.org/10.1016/S0191-8869(00)00113-6

Garnefski, N., Kraaij, V., \& Spinhoven, Ph. (2002). Manual for the use of cognitive emotion regulation questionnaire. Leiderdorp, Netherlands: DATEC.

Geen, R. G. (2001). Human aggression. Philadelphia, PA: Open University Press.

Gratz, K. L., \& Roemer, L. (2004). Multidimensional assessment of emotion regulation and dysregulation: Development, factor structure, and initial validation of the difficulties in emotion regulation scale. Journal of Psychopathology and Behavioral Assessment, 36, 41-54. http://dx.doi.org/10.1023/B:JOBA.0000007455.08539.94

Gratz, K. L., \& Tull, M. T. (2010). Emotion regulation as a mechanism of change in acceptance- and mindfulness-based treatments. In R. A. Baer (Ed.), Assessing mindfulness and acceptance: Illuminating the processes of change. Oakland, CA: New Harbinger Publications.

Greenberg, L. S., Elliott, R., \& Pos, A. E. (2007). Emotion-focused therapy: An overview. European Psychotherapy, 7(1), 19-39. http://dx.doi.org/10.1.1.458.7814

Gross, J. J. (2002). Emotion regulation: Affective, cognitive, and social consequences. Psychophysiology, 39, 281-291. http://dx. doi.org/10.1017/S0048577201393198

Gross, J. J., \& Thompson, R. A. (2007). Handbook of emotion regulation. New York, NY: Guilford Press; US.

Harrington, N. (2005). The frustration discomfort scale: Development and psychometric properties. Clinical Psychology \& Psychotherapy, 12(5), 374-387. http://dx.doi.org/10.1002/cpp.465

Harrington, N. (2006). Frustration intolerance beliefs: Their relationship with depression, anxiety, and anger, in a clinical population. Cognitive Therapy and Research, 30(6), 699-709. http://dx.doi. org/10.1007/s10608-006-9061-6

Harrington, N. (2007). Frustration intolerance as a multidimensional concept. Journal of Rational-Emotive \& Cognitive-Behavior Therapy, 25(3), 191-211. http://dx.doi.org/10.1007/s10942-0060051-0

Hayes, S. C., \& Hofmann, S. G. (2018). Survival circuits and therapy: From automaticity to the conscious experience of fear and anxiety. Current Opinion in Behavioral Sciences, 24, 21-25. http://dx.doi.org/10.1016/j.cobeha.2018.02.006

Hu, L., \& Bentler, P. M. (1999). Cutoff criteria for fit indexes in covariance structure analysis: Conventional criteria versus new alternatives. Structural Equation Modeling: A Multidisciplinary Journal, 6(1), 1-55. http://dx.doi.org/10.1080/10705519909540118

Jermann, F., Van der Linden, M., d'Acremont, M., \& Zermatten, A. (2006). Cognitive emotion regulation questionarie (CERQ): Confirmatory factor analysis and psychometric properties of the french traslation. European Journal of Psychological Assessment, 22(2), 126-131. http://dx.doi.org/10.1027/1015-5759.22.2.126
Jibeen, T. (2012). Frustration intolerance beliefs as predictors of emotional problems in university undergraduates. Journal of Rational-Emotive \& Cognitive-Behavior Therapy, 31(1), 16-26. http://dx.doi.org/10.1007/s10942-012-0154-8

Jibeen, T. (2016). Unconditional self acceptance and self esteem in relation to frustration intolerance beliefs and psychological distress. Journal of Rational-Emotive \& Cognitive-Behavior Therapy, 35(2), 207-221. http://dx.doi.org/10.1007/s10942-016-0251-1

Lei, M., \& Lomax, R. G. (2005). The effect of varying degreesof nonnormality in structural equation modeling. Structural Equation Modeling: A Multidisciplinary Journal, 12(1), 1-27. http://dx.doi. org/10.1207/s15328007sem1201_1

Lorenz, K. (1966). Historia natural de la agresión. España: Siglo XXI Editores.

Martin, R. C., \& Dahlen, E. R. (2005). Cognitive emotion regulation in the prediction of depression, anxiety, stress, and anger. Personality and Individual Differences, 39(7), 1249-1260. http:// dx.doi.org/10.1016/j.paid.2005.06.004

Mauss, I. B., Bunge, S. A., \& Gross, J. J. (2007). Culture and automatic emotion regulation. In S. Ismer, S. Jung, S. Kronast, C. van Scheve \& M. Vanderkerckhove (Eds.), Regulating emotions: Culture, social necessity and biological inheritance. London: Blackwell Publishing.

Mauss, I. B., Cook, C. L., \& Gross, J. J. (2007). Automatic emotion regulation during an anger provocation. Journal of Experimental Social Psychology, 43, 698-711. http://dx.doi.org/10.1016/j. jesp.2006.07.003

McLaughlin, K. A., Hatzenbuehler, M. L., Mennin, D. S., \& Nolen-Hoeksema, S. (2011). Emotion dysregulation and adolescent psychopathology. Behavior Research and Therapy, 49, 544-554. http://dx.doi.org/10.1016/j.brat.2011.06.003

Medrano, L., Franco, P., \& Mustaca, A. (2018). Adaptación argentina de la escala de intolerancia a la frustración. Behavioral Psychology/Psicología Conductual, 26(2), 303-321.

Medrano, L. A., Moretti, L., Ortiz, A., \& Pereno, G. (2013). Validación del cuestionario de regulación emocional cognitiva en universitarios de Córdoba, Argentina. Psykhe, 22(1), 83-96. http://dx.doi.org/10.7764/psykhe.22.1.473

Montero, I., \& León, O. (2007). A guide for naming research studies in psychology. International Journal of Clinical and Health Psychology, 7(3), 847-862.

Mustaca, A. (2013). "Siento un dolor en el alma": ¿metáfora o realidad? Revista Argentina de Ciencias del Comportamiento, 5(2), 47-60. http://dx.doi.org/10.30882/1852.4206.v5.n2.5147

Mustaca, A. (2018). Frustración y conductas sociales. Avances en Psicología Latinoamericana, 36(1), 65-81. http://dx.doi. org/10.12804/revistas.urosario.edu.co/apl/a.4643

Mustaca, A., Martínez, C., \& Papini, M. R. (2000). Surprising nonreward reduces aggressive behavior in rats. International Journal of Comparative Psychology, 13(1), 91-100. Recuperado de https://escholarship.org/uc/item/1qt6d2sf.

Potegal, M., \& Stemmler, G. (2009). Cross-disciplinary views of anger: Consensus and controversy. International Handbook of Anger, 3-7. http://dx.doi.org/10.1007/978-0-387-89676-2_1

Roberton, T., Daffern, M., \& Bucks, R. (2012). Emotion regulation and aggression. Aggression and Violent Behavior, 17, 72-82. http://dx.doi.org/10.1016/j.avb.2011.09.006

Rodríguez, C. M., Baker, L. R., Pu, D. F., \& Tucker, M. C. (2017). Predicting parent-child aggression risk in mothers and fathers: Role of emotion regulation and frustration tolerance. Journal of Child and Family Studies, 26(9), 2529-2538. http://dx.doi. org/10.1007/s10826-017-0764-y

Roll, J., Koglin, U., \& Petermann, F. (2012). Emotion regulation and childhood aggression: Longitudinal associations. Child Psychiatry and Human Development, 43, 909-923. http://dx.doi. org/10.1007/s10578-012-0303-4 
Ruddle, A., Pina, A., \& Vásquez, E. (2017). Domestic violence offending behaviors: A review of the literature examining childhood exposure, implicit theories, trait aggression and anger rumination as predictive factors. Aggression and Violent Behavior, 34, 154-165. http://dx.doi.org/10.1016/j.avb.2017.01.016

Schneider, W., \& Shiffrin, R. M. (1977). Controlled and automatic human information processing I: Detection, search and atention. Psychological Review, 84, 1-66. http://dx.doi.or$\mathrm{g} / 10.1037 / 0033-295 X .84 .1 .1$

Selby, E. A., Anestis, M. D., \& Joiner, T. E. (2008). Understanding the relationship between emotional and behavioral dysregulation: Emotional cascades. Behaviour Research and Therapy, 46(5), 593-611. http://dx.doi.org/10.1016/j.brat.2008.02.002

Skinner, B. F. (1953). Science and Human Behavior. Nueva York: McMillan.

Stanković, S., \& Vukosavljević-Gvozden, T. (2011). The relationship of a measure of frustration intolerance with emotional dysfunction in a student sample. Journal of Rational-Emotive \& Cognitive-Behavior Therapy, 29(1), 17-34. http://dx.doi.org/10.1007/ s10942-011-0128-2

Tager, D. T., Good, G. E., \& Brammer, S. (2010). Walking over 'em’: An exploration of relations between emotion dysregulation, masculine norms, and intimate partner abuse in a clinical sample of men. Psychology of Men and Masculinity, 11, 233-239. http://dx.doi.org/10.1037/a0017636

Tripaldi, S., Paparusso, M., Amabili, M., Manfredi, C., Caselli, G., Scarinci, A., ..., \& Mezzaluna, C. (2018). Frustration discomfort scale (FDS). A psychometric study of the Italian version. Journal of Rational-Emotive \& Cognitive-Behavior Therapy, 36(3), 267287. http://dx.doi.org/10.1007/s10942-018-0286-6
Tull, M. T., Jakupcak, M., Paulson, A., \& Gratz, K. L. (2007). The role of emotional inexpressivity and experiential avoidance in the relationship between posttraumatic disorder symptom severity and aggressive behavior among men exposed to interpersonal violence. Anxiety, Stress and Coping, 20, 337-351. http://dx.doi. org/10.1080/10615800701379249

Werner, K. W., \& Gross, J. J. (2009). Emotion regulation and psychopathology: A conceptual framework. In A. Kring \& Sloan (Eds.), Emotion regulation and psychopathology. New York: The Guilford Press.

Wiedeman, A. M., Black, J. A., Dolle, A. L., Finney, E. J., \& Coker, K. L. (2015). Factors influencing the impact of aggressive and violent media on children and adolescents. Aggression and Violent Behavior, 25, 191-198. http://dx.doi.org/10.1016/j. avb.2015.04. 008

Williams, L. E., Bargh, J. A., Nocera, C. C., \& Gray, J. R. (2009). The unconscious regulation of emotion: Nonconscious reappraisal goals modulate emotional reactivity. Emotion, 9, 847-854. http://dx.doi.org/10.1037/a0017745

Wranik, T., \& Scherer, K. R. (2010). Why do i get angry? A componential appraisal approach. In M. Potegal, G. Stemmler \& C. Spielberger (Eds.), International handbook of anger. Constituent and concomitant biological, psychological, and social processes (pp. 243-267). New York: Springer. 